Principles of Plasma Discharges and Materials Processing,

\section{2nd Edition}

Michael A. Lieberman and

Allan J. Lichtenberg

(John Wiley \& Sons, 2005)

757 pages; $\$ 115.00$

ISBN 0-471-72001-1

The authors have done an excellent job of clearly explaining the different nomenclature used in plasma processing, with the meaning of the symbols and constants commonly encountered. The book appears to be aimed at graduate students and has an extensive mathematical treatment of plasma processes. As the authors have mentioned, much of the content of the book is based on the courses they have taught over the years. Their focus has been on semiconductor thin-film applications for the microelectronics processing industries and this has been well covered. The use of problem sets at the end of each chapter is very useful from an academic perspective. It is very well written for those who specialize in this field although too detailed for average practitioners in the field. It would have been useful for practitioners if it offered examples of the effect of process parameters on real issues (e.g., preferential etch rates between metal and photoresist layers at different lateral dimensions). It is recognized that the geometry of the particular vacuum chamber is crucial to derive this; nevertheless, data could have been collected from published literature with appropriate acknowledgment and references.

The authors seem to have neglected coverage of plasma technology as related to the use of ion beams, namely, ionbeam etching, reactive ion-beam etching, chemical-assisted ion-beam etching, and ion-beam deposition. These processes are extensively used in the manufacture of multilayer device structures used in the disk drive industry and should have been included as a separate chapter. As the demand for data storage-for example, personal handheld devices-increases, there is a concomitant need to keep the size of the gadgets small. This in turn puts a tremendous demand on the physical size of the hard drives, thereby making the manufacture of read and write heads challenging. The role of ion-beam technology and the understanding of the plasma behavior to focus these ion beams for etching and deposition also become very important.

Overall, this is a good book for an academic course that will provide the foundation to senior-level and graduate students inclined to pursue a career in plasma engineering and technology.

Reviewer: Sudhi Sant is president and founder of Twin Technologies Inc., Garden Grove, California and has over 15 years of thin-film deposition and etching experience covering a range of applications.

\section{Nanophysics and Nanotechnology: An Introduction to Modern Concepts in Nanoscience Edward L. Wolf \\ (Wiley-VCH, 2004) \\ 174 pages, $\$ 89.00$ \\ ISBN 3-527-40407-4}

Nanotechnology is a broad subject with many interdisciplinary facets. For the uninitiated, it is best to approach the subject from the foundation of a base discipline. Wolf provides such an introduction from the physics perspective in his new, well-written textbook, Nanophysics and Nanotechnology.

The text surveys the fast-paced topic for

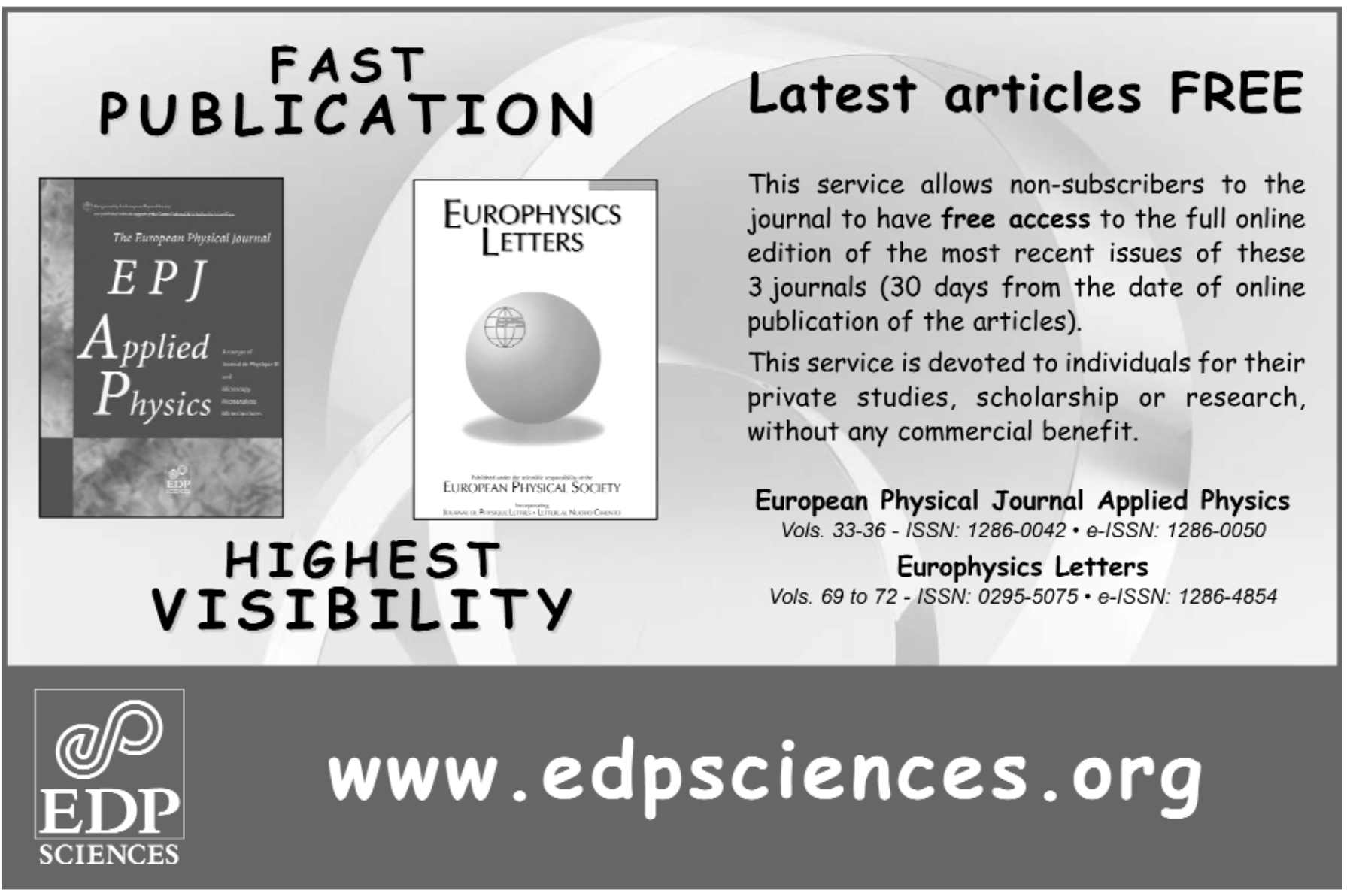

For more information, see http://advertisers.mrs.org 
undergraduate students having a prerequisite background in introductory physics and chemistry. Wolf provides a nice introduction to nanophysics by way of many real-world (natural and synthetic) examples of nanoscale phenomenology. At the outset, the text provides clear motivations for the growing interest in nanotechnology, and then later expands on the science and technology in detail. Background science tutorials and homework problems are woven in throughout the text.

The book delivers its information in three main pieces. The first is an introduction to the "big picture" of nanophysics and nanotechnology, providing motivation for the intense research activity in the field, describing scaling laws and limits to smallness. Starting the text this way is valuable to an interdisciplinary audience needing a quick overview. Since the nanoscale regime represents the crossover region from classical to quantum physics, the second portion is focused on quantum phenomena in nanoscale systems, including a good introduction to quantum physics. The last piece provides information on the fabrication methods and selfassembly of nanostructures. It is refreshing to see that Wolf provides both physical and biological examples of interesting functional nanostructures.

Nanophysics and Nanotechnology contains more pedagogical physics but has a somewhat smaller range of phenomenology than does the book Introduction to Nanotechnology (Wiley, 2003) by Poole and Owens. Faculty in the process of selecting a textbook would be well advised to compare the two to match course objectives.

The subject of nanotechnology presents a challenge: any textbook (this one included) on the fast-moving field of nanotechnology will be somewhat out of date the day it hits the shelves. The same has been true in the field of solid-state physics. (For example, consider the many editions of Charles Kittel's Introduction to Solid State Physics.) Nonetheless, a good introductory text provides a foundation in concepts and terminology for beginners so that they acquire the requisite tools to read and understand the research literature. Wolf's book goes a long way toward accomplishing that goal. Ultimately, the book provides a clear introduction to nanophysics for the beginning student or for interdisciplinary researchers needing a crash course in nanophysics.

Reviewer: Mark Tuominen is a professor of physics and co-director of MassNanoTech at the University of Massachusetts Amherst. Tuominen's experimental research emphasizes nanofabrication, nanomagnetics, and nanoscale charge transport phenomena.

\section{Acetylene Chemistry: Chemistry, Biology, and Material Science François Diederich, Peter J. Stang, and Rik R. Tykwinski, eds. \\ (John Wiley \& Sons, 2005) \\ 528 pages; $\$ 185.00$ \\ ISBN 3-527-30781-8}

Acetylene chemistry is an important field of chemistry for me since most of the molecules synthesized in our molecular electronics and nanomachines programs contain alkynyl units. Therefore, I reviewed this important book with a great deal of personal interest. I was not disappointed, as its 11 chapters, each with 60-200 references, is generally a broad treatment of the field. While some chapters were unfortunately reviews of the chapter author's own work with little overview of the work of others, most chapters nicely covered the breadth of the titled topic. Each chapter, except for the first, which covers theoretical studies, and the ninth, which mainly covers spectroscopy of carbon clusters, contains an in-depth discussion of a particular subfield of acetylenic chemistry along with representative synthetic methods - a nice feature for the researcher seeking an assessment of the synthetic prowess needed for a particular transformation. For instance, Larock covers synthesis of heterocycles and carbocycles by electrophilic cyclization of alkynes; Ashwanden and Carreira review the addition of terminal acetylides to aldehydes, ketones, imines, and nitrones; and Swager reviews the synthesis of semiconducting poly(arylene ethylene)s. The text is well illustrated, with some figures in color, and a 14-page index affords one the ability to search for specific terms. I recommend this book to those interested in synthesis of acetylenic molecules or in synthesis of advanced compounds from alkynyl starting materials. The book would also be a good resource for use in a graduate-level course on alkyne synthesis. In addition, this book provides the synthetic tools for the preparation of a class of compounds of widespread interest to materials scientists.

Reviewer: James M. Tour is the Chao Professor of Chemistry at Rice University in the Chemistry Department and Center for Nanoscale Science \& Technology.

\section{Path Integrals in Quantum Mechanics, Statistics, Polymer Physics, and Financial Markets, 3rd Edition \\ Hagen Kleinert \\ (Imperial College Press, 2004) \\ 1504 pages; $\$ 138.00$ \\ ISBN 981-238-106-6}

This book is the third edition of Kleinert's monograph on path integrals, which first appeared in 1990. Since their development by Feynman, based on early ideas from Dirac, path integrals have blossomed into one of the central tools in theoretical physics and chemistry. Path integral approaches have contributed to fields as diverse as quantum fluids, quantum electrodynamics, polymer physics, and timedependent statistical mechanics. And as shown in the current edition of this book, they have found applications far outside physics in modeling stochastic behavior in financial markets. Kleinert gives a comprehensive overview of the wide range of problems that can be addressed with path integration. The book is encyclopedic in its scope, not recommended as an introduction for those first learning path integral methods, but rather as a clear and thorough monograph-a treasure trove of interesting and useful results.

The book starts with a concise summary of basic results in classical, quantum, and statistical mechanics. Path integrals are then developed and analytical solutions are given for model problems such as the free particle and harmonic oscillator. Kleinert's work on variational perturbation theory with Feynman is showcased; there is no better or more complete reference to these useful techniques for estimating free energies without invoking a full path integral representation. A major portion of the book is devoted to Kleinert's work on solving the path integral for the hydrogen atom, one problem that eluded Feynman. There are many other nuggets in the book: semiclassical approximations and the Thomas-Fermi theory, second quantization, nonequilibrium statistical mechanics and Green's functions, and applications in polymer physics. Several additions to chapters in previous editions have extended and clarified major points.

The final chapter in the book on financial markets is new in the third edition. It is a high-level introduction to stochastic methods and path integrals applied to market fluctuations. Kleinert starts with a Gaussian model for market fluctuations but then shows that real markets exhibit heavy tails in their distributions. He proceeds to give an introduction to Levy and other non-Gaussian distributions which can accurately reproduce market data. The Fokker-Planck equation for the evolution of the probability distribution with time is derived from the path integral representation. The theory of martingales is developed, and finally, Kleinert discusses option pricing and the famous Black-Scholes model.

I highly recommend this book as a reference in graduate courses in theoretical 
physics and chemistry, where the student can gain an in-depth understanding of concepts introduced at a more pedagogic level. It is also a valuable reference for researchers in all areas of theoretical physics and chemistry. The material is at a high level but is clearly presented. There are many valuable physical insights throughout the book, and it is well worth the effort to work through Kleinert's sometimes formidable analytical presentations. Path integrals are now part of the language of theoretical physics and stochasticswith the original text by Feynman and Hibbs and this encyclopedic volume on the shelf, readers can gain a deep understanding of the pervasive role of path integrals in representing our physical world.

Reviewer: Thomas Beck is a professor of chemistry and physics at the University of Cincinnati. His research interests relate to the chemical physics of condensed phase systems, including solvation phenomena, quantum effects, electronic structure, transport, and biological ion channels.

\section{Nobel Laureates and Twentieth- Century Physics Mauro Dardo \\ (Cambridge University Press, 2005) \\ 533 pages; $\$ 39.95$ \\ ISBN 0-521-54008-9}

December 10, 2001, was the 100th anni- versary of the establishment of the Nobel Prizes. Since they were created, 758 Nobel Prizes have been awarded through 2003, 171 of them in physics. This book is directed at scientists, students, and general readers who have an interest in the development of physics during this time period and in the specific contributions of the physics Laureates. Part I (three chapters) presents the background of the development of physics prior to the establishment of the Nobel Prizes. Part II, entitled "New Foundations" (Chapters 4-8), covers the time period from 1901 to 1950; and Part III, entitled "New Frontiers" (Chapters 9-13), covers the period from 1951 to 2003. In these two parts, the presentation is strictly chronological but covers general advances and trends in physics as well as the specific contributions of each of the physics Nobelists.

The main part of the book, Chapters $4-13$, is considerably enriched by the inclusion of illustrations, about 20 per chapter. These range from formal portraits of Nobelists, to scientific pictures of apparatus or experimental results, to schematic drawings, to informal snapshots of physicists at work or play, and to pictures of relevant buildings and places. An approximately 60-page section at the end of the book presents some very use- ful information for the reader: a complete list of the Nobelists in physics with a brief description of the basis for each award, a 300-word glossary of technical terms, a chapter-by-chapter listing of footnotes and literature citations, two bibliographies (selected books and further reading), and a detailed index.

The writing style is clear, entertaining in the inclusion of anecdotes and down-toearth examples, and technically sound. These qualities arise from the interests and experience of the author. Dardo is a professor of experimental physics at the Amedeo Avogadro University at Alessandria, Italy, where he has also served as dean of the faculty of sciences. Apart from his own research in experimental physics, he has had a lifelong interest in history, especially the history of science, and in the diffusion of scientific culture. His experience and enthusiasm have contributed enormously to the appeal of this engaging book. Would that every field of science had just such a literate, knowledgeable, and enthusiastic champion as Dardo!

Reviewer: Jack H. Westbrook is owner of and principal consultant with Brookline Technologies, a consulting firm in Ballston Spa, N.Y., where he consults on materials and technical information systems. He is chair of the MRS Bulletin Book Review Board and serves on the MRS Bulletin Editorial Board.

\begin{tabular}{|c|c|}
\hline $\begin{array}{l}\text { Editorial Board } \\
\text { Pulickel M Ajayan } \\
\text { Valery Alakhov } \\
\text { Chunli Bai } \\
\text { Raj Bawa } \\
\text { Salvatore Cannistraro } \\
\text { Frank Caruso } \\
\text { Joao Pedro Conde } \\
\text { Harold G Craighead } \\
\text { Adam Curtis } \\
\text { Tejal A Desai } \\
\text { Ruth Duncan } \\
\text { Elias Fattal } \\
\text { Hicham Fenniri } \\
\text { Mauro Ferrari } \\
\text { Alexander Florence } \\
\text { Robert A Freitas Jr } \\
\text { Leslie A Geddes } \\
\text { Gershon Golomb } \\
\text { Mark Gumbleton } \\
\text { Alexander V Kabanov } \\
\text { Agnes Kane } \\
\text { Kazunori Kanaoka } \\
\text { Thomas H Kissel } \\
\text { Kostas Kostarelos } \\
\text { Wolfgang G Kreyling } \\
\text { Cato T Laurencin } \\
\text { Kam W Leong } \\
\text { Jean-Christophe Leroux } \\
\text { Kalle Levon } \\
\text { Andrew McCaskie } \\
\text { Carlo D Montemagno } \\
\text { Shuming Nie } \\
\text { Nicholas A Peppas } \\
\text { Molly S Shoichet } \\
\text { S Sridhar } \\
\text { Vicki Stone } \\
\text { Vladimir Torchilin } \\
\text { Karen L Wooley }\end{array}$ & $\begin{array}{l}\text { Contents: Volume I Issue I } \mathbf{2 0 0 6} \\
\text { - Nanopillar array structures for enhancing biosensing performance } \\
\text { - Increased endothelial and vascular smooth muscle cell adhesion on nanostructured titanium and } \\
\text { CoCrMo } \\
\text { - Silk- fibroin-coated liposomes for long-term and targeted drug delivery } \\
\text { - Peptide crosslinked micelles: a new strategy for the design and synthesis of peptide vaccines } \\
\text { - Selective adhesion and mineral deposition by osteoblasts on carbon nanofiber patterns } \\
\text { - Polydisulfide Gd(III) chelates as biodegradable macromolecular magnetic resonance imaging contrast } \\
\text { - Mgents } \\
\text { - Surface functionalization of gold nanoparticles using hetero-bifunctional poly(ethylene glycol) spacer for } \\
\text { - intracellular tracking and delivery } \\
\text { - On the way to building an integrated computational environment for the study of developmental } \\
\text { - Tatterns and genetic diseases } \\
\text { - Whanofibers and their applications in tissue engineering } \\
\text { - Quantum chemical analysis of deferiprone - iron binding reaction } \\
\text { - Incorporation of quantum dots on virus in polycationic solution }\end{array}$ \\
\hline $\begin{array}{l}\text { Editor-in-Chief } \\
\text { Thomas J Webster } \\
\text { Purdue University }\end{array}$ & 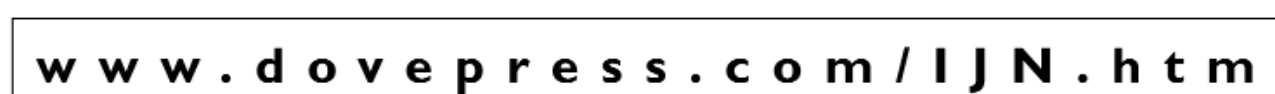 \\
\hline
\end{tabular}

For more information, see http://advertisers.mrs.org 